\title{
Gamma oscillations as a mechanism for selective information transmission
}

\author{
Stan Gielen · Martin Krupa • Magteld Zeitler
}

Received: 20 January 2010 / Accepted: 1 April 2010 / Published online: 27 April 2010

(C) The Author(s) 2010. This article is published with open access at Springerlink.com

\begin{abstract}
In the past decades, many studies have focussed on the relation between the input and output of neurons with the aim to understand information processing by neurons. A particular aspect of neuronal information, which has not received much attention so far, concerns the problem of information transfer when a neuron or a population of neurons receives input from two or more (populations of) neurons, in particular when these (populations of) neurons carry different types of information. The aim of the present study is to investigate the responses of neurons to multiple inputs modulated in the gamma frequency range. By a combination of theoretical approaches and computer simulations, we test the hypothesis that enhanced modulation of synchronized excitatory neuronal activity in the gamma frequency range provides an advantage over a less synchronized input for various types of neurons. The results of this study show that the spike output of various types of neurons [i.e. the leaky integrate and fire neuron, the quadratic integrate and fire neuron and the Hodgkin-Huxley (HH) neuron] and that of excitatory-inhibitory coupled pairs of neurons, like the Pyramidal Interneuronal Network Gamma (PING) model, is highly phase-locked to the larger of two gamma-modulated input signals. This implies that the neuron selectively responds to the input with the larger gamma modulation if the amplitude of the gamma modulation exceeds that of the other signals by a certain amount. In that case, the output of
\end{abstract}

S. Gielen (凶) $\cdot$ M. Krupa $\cdot$ M. Zeitler

Department of Biophysics, Donders Institute for Brain,

Cognition and Information, Radboud University Nijmegen,

Geert Grooteplein 21, 6525 EZ Nijmegen, The Netherlands

e-mail: S.Gielen@donders.ru.nl

M. Krupa

e-mail: M.Krupa@donders.ru.nl

M. Zeitler

e-mail: M.Zeitler@donders.ru.nl the neuron is entrained by one of multiple inputs and that other inputs are not represented in the output. This mechanism for selective information transmission is enhanced for short membrane time constants of the neuron.

Keywords Synchronization · Gamma oscillations · Phase locking $\cdot$ Stimulus selection

\section{Introduction}

Action potentials, or spikes, are responsible for transmitting information through the nervous system (Adrian 1932). In the past decades, many experimental and theoretical studies have focussed on the relation between spike input and output of neurons and of populations of neurons with the aim to understand information processing by (groups of) neurons (see, e.g. Tiesinga et al. 2001; Dayan and Abbott 2001; Escalona et al. 2002; Kuhn et al. 2004; Herz et al. 2006). In general, most of these studies have investigated the response of a neuron to a single stimulus, which may be encoded in single-unit or multi-unit activity. This means that if multiunit activity was studied as input to a neuron, the multi-unit activity represented one signal, such as for a population of Poisson neurons, all modulated by the same signal. Several measures, such as Mutual Information, Fischer information and coherence (for an overview, see, e.g. Averbeck et al. 2006), have been used to quantify the amount of information in the input and output. These studies have provided a thorough overview of the neuronal properties and of the neural encoding of information that is critical for reliable information transmission (see, e.g. Meunier and Segev 2001; Hansel et al. 1995).

A particular aspect of neuronal information, which has not received much attention so far, concerns the problem of 
information transfer when a neuron or a population of neurons receives input from two or more (populations of) neurons, in particular when these (populations of) neurons carry different types of information. This might happen, for example, when two stimuli are presented in the receptive field of a neuron in the visual system. The question then arises as to whether the output of the neuron reflects a linear summation of the information contained in both inputs, or whether one input signal may dominate over the other. A good example where this is relevant is in selective attention (Reynolds and Desimone 2003), where two stimuli are presented within the receptive field of a single neuron. Experimental studies in visual cortex (Treue and Maunsell 1996; Luck et al. 1997; Reynolds et al. 1999; Reynolds and Desimone 2003) have shown that the output of a neuron may reflect either input, depending on whether attention is directed to one or the other stimulus. This biasing of competition in favour of the attended stimulus is correlated with the appearance of enhanced gamma band $(30-80 \mathrm{~Hz})$ synchronization (Fries et al. 2001, 2007; Fries 2009; Gruber et al. 1999; Taylor et al. 2005; Womelsdorf et al. 2005). This observation suggests that the responses of a neuron to multiple input signals not only depend on the properties of the neuron, but also on the neuronal encoding of information in oscillatory, synchronized firing of the spike input.

Recently, Fries (2005), Fries et al. (2007) and Börgers and Kopell (2008) hypothesized a specific link between gamma rhythmicity in neuronal firing and the selective transmission of information. These authors postulated that a coherent input oscillating in the gamma frequency range can be highly effective at preventing another less coherent input to affect the output of a neuron. This hypothesis implies that selective information transmission is determined to a large extent by the amount of synchronized, oscillatory neuronal activity at the input. Two factors are thought to contribute to this effect. The first is that balanced excitation and inhibition decrease the resistance of the cell membrane and thereby raise the leakiness of the target neurons. If the time constant of the cell membrane decreases, the neuron behaves more like a coincidence detector and tends to respond better to synchronized input (Azouz and Gray 2000; Kuhn et al. 2004), thereby greatly amplifying the advantage of a more coherent excitatory input $A$ over a less coherent competitor $B$. The second factor relates to the role of inhibition (Börgers et al. 2010). One way to generate periodic inhibition is by the "PING" (Pyramidal Interneuronal Network Gamma) mechanism (Börgers and Kopell 2003, 2005; Whittington et al. 2000), in which a gamma rhythm arises from the interaction between excitatory pyramidal cells (E-cells) and inhibitory interneurons (I-cells). A synchronized gamma frequency train $A$ of excitatory input pulses triggers synchronous spike volleys of the I-cells, which then synchronize the E-cells by inhibitory spike volleys. The timing of the inhibition from the inhibitory neurons after the spike volleys of a coherent stimulus A to the excitatory neurons decreases the contribution of a competing, less coherent pulse train $B$ that is uncorrelated with the more coherent stimulus $A$. As demonstrated by Börgers and Kopell (2008), this inhibition amplifies the advantage of coherent input by raising the effective leakiness of the target neurons. Therefore, the two factors of leakiness and timing of inhibition are partly related.

Leakiness and inhibition provide modulations in excitability, which are the key for the Communication-throughCoherence (CTC) hypothesis by Fries (2005), which states that oscillatory activity is a mechanism for efficient information transfer. The aim of the present study is to test the hypothesis that enhanced modulation of synchronized excitatory neuronal activity in the gamma frequency range provides an advantage over a less synchronized input for various types of neurons using theoretical approaches and computer simulations. Recently, Börgers and Kopell (2008) have shown that more coherent excitatory stimuli may have a competitive advantage over less coherent ones for coupled excitatory and inhibitory neurons, in agreement with experimental observations. In their study, they investigated the responses of the PING model (consisting of an excitatory neuron and an inhibitory neuron, bi-directionally coupled to each other), to two input signals, each composed of a series of Gaussians at a gamma rhythm. For a small standard deviation $\sigma$ of the Gaussians, the input was sharply peaked, whereas the oscillation amplitude of the input rapidly decreases for broader pulses with increasing $\sigma$. Sharper peaks and higher amplitude correspond to more synchronized input spikes, corresponding to a more coherent input. The main finding of Börgers and Kopell was that the responses to the less coherent signal are suppressed by the more coherent signal as long as the inhibitory neuron provides sufficiently strong and longlasting inhibition after firing of the excitatory neuron.

The main focus of our study is to explore in detail, both analytically and by computer simulations, the responses of various types of single model neurons to multiple gammamodulated input signals. There are two main differences between our work and the work by Börgers and Kopell. The first difference relates to the fact that we focus mainly on the responses of single model neurons. A few simulations were done for the PING model to compare the results for the single neurons to that for the PING model. The second difference is related to the type of input. In the study by Börgers and Kopell (2008), the width and amplitude of the gaussianshaped volleys covaried, whereas we only considered variations in amplitude of the gamma-modulated signal. We use sinusoidal input signals with a frequency in the gamma range superimposed on a mean firing rate, rather than periodic Gaussian-shaped input. Recordings of EEG and MEG (see, e.g. Schoffelen et al. 2005) and local field potentials (see Womelsdorf et al. 2005) have shown that a realistic gamma 
Fig. 1 Phase locking for a LIF neuron with membrane time constant $\tau=7 \mathrm{~ms}$ in the presence of one sinusoidal input (lower panels in $\mathbf{a}$ and $\mathbf{b}$ ) with frequency $\gamma=43 \mathrm{~Hz}$.

a Membrane potential for sinusoidal input with amplitude $B=4.7 \mathrm{~s}^{-1}$. The phase of the sinusoid, when the LIF neuron spikes, decreases when the amplitude $B$ increases. This is illustrated in panel $\mathbf{b}$, where $B=6 \mathrm{~s}^{-1}$. For clarity, dashed lines relate the time of the spike to the sinusoidal input. $\mathbf{c}$ The locking phase as a function of the amplitude $B$ of the sinusoidal input. d The time it takes to the next spike for the LIF neuron given the initial condition $V(0)=0$ and the initial phase equal to $\varphi$. The dashed line corresponds to $B=3.5 \mathrm{~s}^{-1}$, the solid line to $B=4.147 \mathrm{~s}^{-1}$ and the dashed-dotted line to $B=6 \mathrm{~s}^{-1}$ The left intersection point of the dashed-dotted line with the line $T=1000 / 43 \approx 23.25 \mathrm{~ms}$ gives the (stable) locking phase
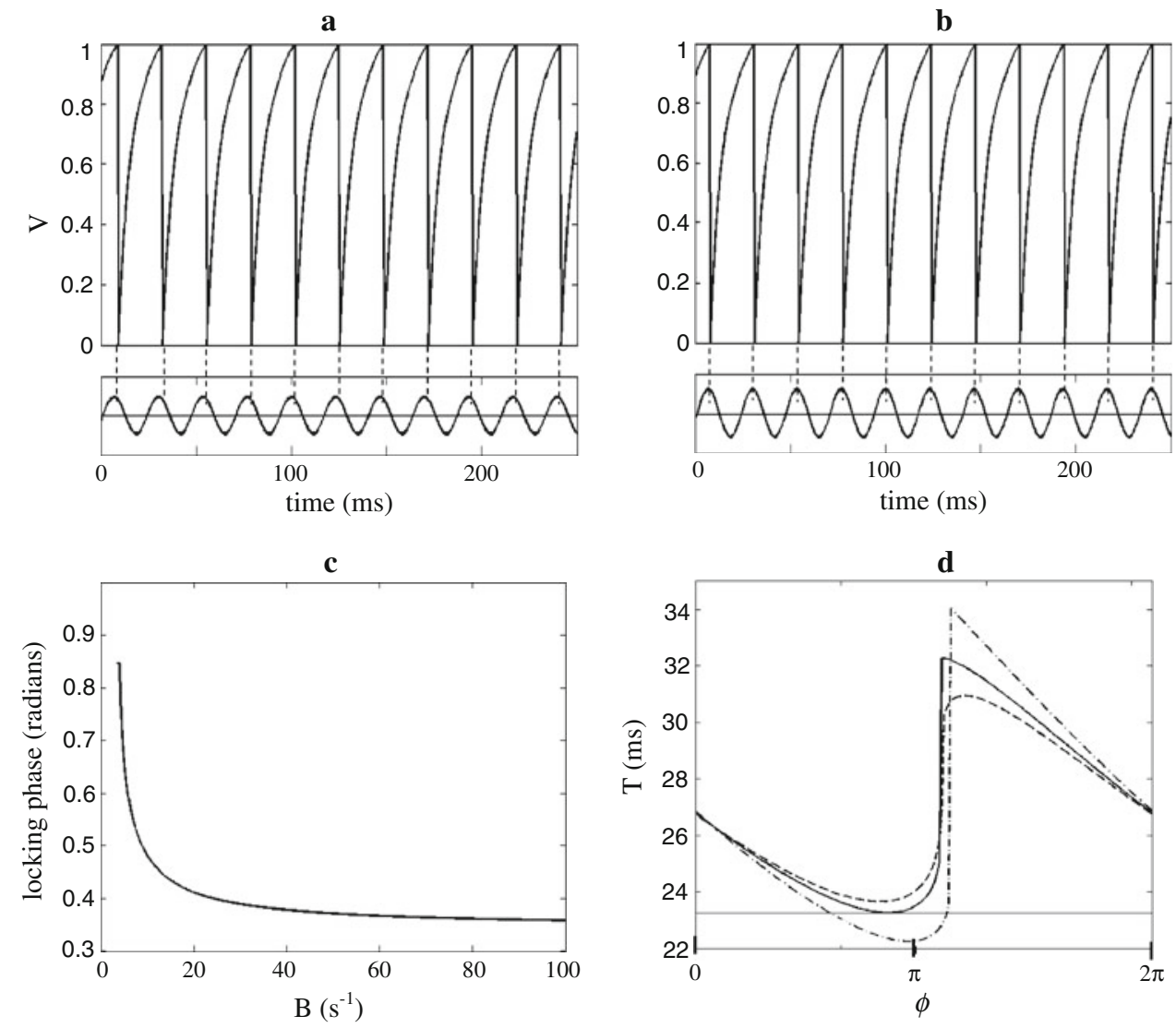

signal has a band-pass frequency spectrum and look very similar to band-pass filtered Gaussian White noise (see Womelsdorf et al. 2005, Fig. 1). A rougher but still reasonable approximation is by a sinusoid, which motivates our choice. Through a combination of theoretical results and simulations, we show that entrainment of the output of the LIF neuron can occur by the input with the larger gamma modulation and we specify the parameter range where it takes place. The combined simplicity of our input signal and the LIF model allows for some rigorous results.

Our results demonstrate that stimulus selection can take place at the level of a single neuron, even without inhibition as in the PING model. The input signal with the larger gamma modulation can entrain the output of a single neuron because the structure of the input prevents firing at specific time windows. If the amplitude of one of the gamma signals is sufficiently large, the neuron may fire at a high probability when the sinusoid is positive, and by the time that the membrane potential returns to the firing threshold again, the sinusoid with the larger amplitude becomes negative and thereby reduces the probability of firing. This result applies to a variety of models [quadratic integrate and fire neuron, Hodgkin-Huxley (HH) neuron, PING model] showing that the pattern of the entrainment of the output of the neuron by the input with a larger gamma modulation is similar as in the case of the LIF neuron.

\section{Phase coherence as measure of entrainment}

In order to study entrainment, we will study the responses of a few types of neurons and pairs of excitatory and inhibitory coupled neurons to an input signal modulated by two different sinusoids at a gamma frequency. A quantitative measure for entrainment of a neuron by sinusoidally modulated inputs can be obtained using the phase coherence, which is defined by $R=\left|\frac{1}{N} \sum_{j=1, N} e^{i 2 \pi \omega_{j} t_{j}}\right|$. Here, the summation is over all $N$ output spikes of the neuron with corresponding firing times $t_{\mathrm{j}}$ and $\omega_{j}$ represents the frequency of the sinusoidal input signal $j$ (Lachaux et al. 1999). The phase coherence provides a measure for the variability of the spike output relative to the phase of the input signal with frequency $\omega_{j}$. It has the value 1 if the neuron perfectly locks to the input signal (i.e. all output spikes are at a fixed phase in the cycle of the input signal). The value is 0 if there is no locking at all, i.e. the spikes are generated at completely random times relative to the periodic input signal. 
Throughout this study, our input consists of one or two gamma signals, represented by sinusoids with frequencies $\omega_{1}$ and $\omega_{2}$, superimposed on a constant input, which ensures a mean firing rate in the absence of the sinusoidal input signals.

\section{LIF neuron}

The dynamics of the linear integrate and fire (LIF) neuron is given by

$\frac{\mathrm{d} V}{\mathrm{~d} t}=-\frac{1}{\tau}\left(V-V_{0}\right)+\frac{I}{C}$,

where $C$ represents the (constant) capacitance of the cell membrane and $\tau=R_{\mathrm{m}} C$ is the time constant of the neuron with $R_{\mathrm{m}}$ representing the membrane resistance. When the voltage $V$ reaches a threshold value $V_{\theta}$, its value is reset to $V_{0}$. In this article, we will use $V_{0}=0, V_{\theta}=1$, and the dimensionless "membrane potential" $V$, which is scaled to values between 0 and 1 . The parameter $I$ represents the external input to the neuron. Without the reset mechanism, the membrane potential of the neuron has a steady-state value $V_{\infty}=V_{0}+\frac{\tau I}{C}$. If the reset is taken into account, the transition from non-spiking to spiking occurs when the value of $V_{\infty}$ exceeds $V_{\theta}$. We define $I_{b}$ as the transitional value for the input that causes spiking when $V_{\infty}$ exceeds $V_{\theta}$. This transitional input value is given by $I_{b}=C \frac{V_{\theta}-V_{0}}{\tau}$.

In our analysis, the total input to the LIF neuron is defined by

$I / C=\mu+B_{1} \cos \left(2 \pi \gamma_{1} t\right)+B_{2} \cos \left(2 \pi \gamma_{2} t\right)$,

where $B_{1}$ and $B_{2}$ are the amplitudes of two sinusoidal inputs with frequencies $\gamma_{1}$ and $\gamma_{2}$ and where $\mu$ represents a constant input. In order to investigate the effect of the leak time constant $\tau$ of the LIF neuron, simulations were done for different values of $\tau$. Throughout this section, we set the constant input $\mu$ to a value (slightly) larger than $\frac{I_{b}}{C}$ such that the firing rate of the LIF neuron is 38 spikes/s in the absence of any other input signals.

\subsection{Phase-locking to a single sinusoidal input}

For an understanding of the two-input case, it is helpful to first consider the case of a single input:

$I / C=\mu+B \cos (2 \pi \gamma t)$.

More specifically, we consider Eq. 1 with the input current given by Eq. 3 and initial condition $V\left(t_{0}\right)=0$. For this case, the explicit solution for Eq. 1 is given by (see Appendix 1A)

$$
\begin{aligned}
V\left(t+t_{0}\right)= & \left(\tau \mu+V_{0}\right)\left(1-e^{-\frac{t}{\tau}}\right) \\
& -B \frac{\tau}{\sqrt{4 \pi^{2} \gamma^{2} \tau^{2}+1}} \sin \left(2 \pi \gamma t_{0}-\theta\right) e^{-\frac{t}{\tau}} \\
& +B \frac{\tau}{\sqrt{4 \pi^{2} \gamma^{2} \tau^{2}+1}} \sin \left(2 \pi \gamma t_{0}-\theta+2 \pi \gamma t\right)
\end{aligned}
$$

with $\theta=\arctan (2 \pi \gamma \tau)$. In order to obtain an expression for the one-to-one phase-locked solution for driving frequency $\gamma$, we substitute $T=1 / \gamma$ for $t$ and the threshold for firing $V_{\theta}$ for $V\left(\gamma^{-1}+t_{0}\right)$. For simplicity, we define $\varphi=2 \pi \gamma t_{0}$ as the phase of the sinusoidal input at the time when the LIF neuron fires. After some algebra (we refer to Appendix 1A for details), this gives the expression

$B \sin (\varphi-\theta)=\left(\mu_{\gamma}-\mu\right) \sqrt{4 \pi^{2} \gamma^{2} \tau^{2}+1}$,

where $\mu_{\gamma}$ is the constant drive needed for tonic spiking of the LIF neuron at frequency $\gamma$ when $B=0$. Equation 4 gives an explicit expression of the phase $\varphi$ of the sinusoidal input when the LIF neuron fires. We now see that the smallest value of $B$ for phase locking to exist is

$B_{\text {bif }}=\left(\mu_{\gamma}-\mu\right) \sqrt{4 \pi^{2} \gamma^{2} \tau^{2}+1}$

and the corresponding value of $\varphi$ is $\varphi=\theta+\pi / 2$. This shows that the LIF neuron will only synchronize to the sinusoidal input if the amplitude of the sinusoidal input is sufficiently large. For any $B>B_{\text {bif }}$, Eq. 4 has two solutions, one with $\theta<\varphi<\theta+\pi / 2$ and the other with $\pi / 2+\theta<\varphi<\theta+\pi$. A lengthy but straightforward computation shows that it is the smaller value of $\varphi$ which corresponds to stable phase locking (see Appendix 1B). Hence $\varphi$ is between $\theta$ and $\theta+\pi / 2$, and as $B$ increases $\varphi$ decreases towards $\theta$.

Figure 1a and $\mathrm{b}$ shows the membrane potential of the leaky integrate and fire neuron for $\gamma=43 \mathrm{~Hz}$ and $\tau=7 \mathrm{~ms}$ for two values of $B, 4.7 \mathrm{~s}^{-1}$ and $6 \mathrm{~s}^{-1}$, respectively. In both cases, the neuron fires at a more-or-less constant phase of the input signal. This phase is well above the lower bound $\theta=\arctan (2 \pi \gamma \tau)=\arctan (2 \pi \times 43 \times 0.007) \approx \pi / 3$. Note that we use seconds as a unit of time rather than milliseconds. Notice that the phase of the sinusoid at the time of the spike is smaller in Fig. 2b than in Fig. 2a. This illustrates that the phase of firing decreases when the amplitude $B$ of the gamma frequency increases.

We point out that when $B$ is sufficiently large, a solution for Eq. 1 may no longer correspond to a spiking solution with frequency $\gamma$. This happens when the positive phase of the sinusoid generates two spikes in some of the cycles. This is illustrated in Fig. 2, which shows the membrane potential when the amplitude B of the sinusoidal input is just below the value (Fig. 2a) or just above the value (Fig. 2b), where it would generate multiple spikes per cycle. In the latter case, the coherence decreases since spikes are generated at different phases of the sinusoidal input. When the value of $B$ 
Fig. 2 Membrane potential of the LIF neuron (upper panels) and sinusoidal input (lower panels) for the phase-locked solution for a relatively large values of $B$. In a, the locking is almost perfect

( $B=112 \mathrm{~s}^{-1}, \tau=7 \mathrm{~ms}$ and $\gamma=43 \mathrm{~Hz}$ ). If the value $B$ increases any further (see b), a second spike develops causing doublets of firing and a perfect phase-locked solution no longer exists

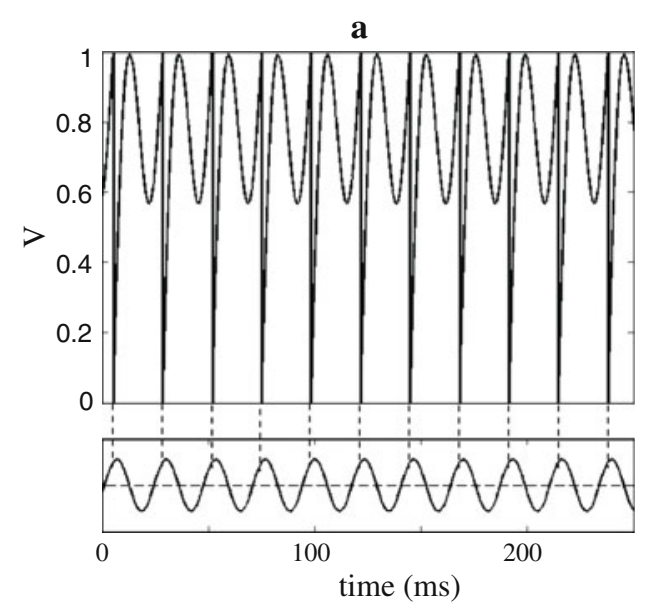

$\mathbf{b}$

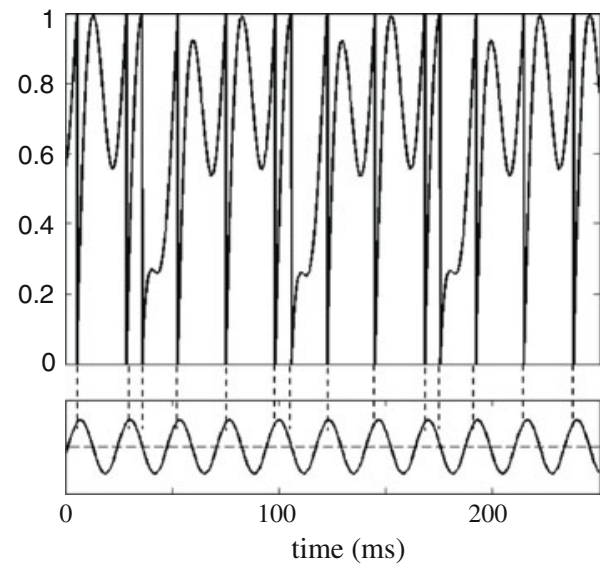

approaches the value, which causes two spikes per cycle, the phase $\varphi$ of the sinusoid at the time of the first spike in the doublet converges to $\theta$. We will not consider this situation for large values of B any further in this article.

Figure 1c shows the dependence of the locking phase $\varphi$ on the amplitude of the sinusoidal input $B$ for $\gamma=43 \mathrm{~Hz}$ and $\tau=$ $7 \mathrm{~ms}$. The value of the locking phase decreases from the initial value $\theta+\pi / 2$, which is approximately $5 \pi / 6$, and asymptotically approaches the value $\theta=\arctan (2 \pi \gamma \tau) \approx \pi / 3$.

In order to further illustrate these results, we computed the inter-spike interval $T(\varphi)$ between a spike at phase $\varphi$ of the sinusoid and the next spike, as a function of $\varphi$. Strictly speaking, this relation is equivalent to the return map $\Pi(\varphi)=\varphi+$ $2 \pi \gamma T(\varphi)(\bmod 2 \pi)$, which gives the phase of the next spike as a function of the phase of a spike. Figure 1d shows $T(\varphi)$ for three values of $B: B<B_{\text {bif }}, B=B_{\text {bif }}$ and $B>B_{\text {bif }}$. If $B<B_{\text {bif }}$ (dashed line), the time interval to the next spike always exceeds the period of the $43 \mathrm{~Hz}$ sinusoid $(23.25 \mathrm{~ms})$. Therefore, phase locking does not occur. When $B=B_{\text {bif }}$ (solid line in Fig. 1d), there is exactly one phase $\varphi$ with $T=1 / \gamma$. If the amplitude of the sinusoidal input exceeds $B_{\text {bif }}$ (dashed-dotted line), there are two intersection points with the horizontal line $T=1 / \gamma=23.25 \mathrm{~ms}$. Only the first value corresponds to a stable state (see Appendix 1B). This value decreases for larger values of $\mathrm{B}$, approaching the lower bound $\theta=\arctan (2 \pi \gamma \tau)=\arctan (2 \pi \times 43 \times 0.007) \approx \pi / 3$ for large values of $\mathrm{B}$.

\subsection{Phase-locking for two sinusoidal inputs}

It turns out that $B_{\text {bif }}$ is also a key parameter for the case with two sinusoidal input signals. The results in Fig. $3 a$ and $b$ show the phase coherence between firing of the LIF neuron and each of the two sinusoidal inputs as a function of the amplitudes $B_{1}$ and $B_{2}$ of the sinusoids. These amplitudes, $B_{1}$ and $B_{2}$, were chosen such that the total input to the LIF neuron was never less than zero. This was achieved by

$B_{1} \leq \frac{3}{2} B_{\text {bif }}\left(\gamma_{1}, \tau\right), \quad B_{2} \leq \frac{3}{2} B_{\text {bif }}\left(\gamma_{2}, \tau\right)$,

Note that this also implies that the average input remains the same as the amplitude of the sinusoid is varied. The values of the gamma frequencies in Fig. 3 are $\gamma_{1}=40 \mathrm{~Hz}$ and $\gamma_{2}=43 \mathrm{~Hz}$.

The value of the phase coherence between the signal with frequency $\gamma_{1}$ and the spike output of the LIF neuron is close to one in the upper left part of Fig. 3a (i.e. for large values of $B_{1}$ and small values of $B_{2}$ ). Similarly, the value of the phase coherence between the signal with frequency $\gamma_{2}$ and the spike output of the LIF neuron is close to one in the lower right part of Fig. $3 \mathrm{~b}$ (i.e. for large values of $B_{2}$ and for small values of $B_{1}$ ). This result shows that the LIF neuron can be entrained by either one of the two sinusoids. If $B_{1}=0 \mathrm{~s}^{-1}$ phase locking to the sinusoidal input with frequency $\gamma_{2}$ starts at $B_{2}=B_{\text {bif }}\left(\gamma_{2}\right)$ (i.e. near $B_{2}=4 \mathrm{~s}^{-1}$ ) and persists for larger values of $B_{2}$. Similarly, phase locking to signal 1 starts at $B_{1}=B_{\text {bif }}\left(\gamma_{1}\right)$ when $B_{2}=0 \mathrm{~s}^{-1}$.

Furthermore, we claim that for $B_{1}>0 \mathrm{~s}^{-1}$, there is approximate phase locking to signal 2 with high coherence values as long as the amplitudes $\left(B_{1}, B_{2}\right)$ are to the right of the line $\left(B, B+B_{\text {bif }}\left(\gamma_{2}\right)\right)$. The claim is based on the following argument, which takes into account not only the amplitudes of the two sinusoids, but also the relative phase, which, because of the small difference in the frequencies, evolves slowly. We treat the case of $B_{1}>0 \mathrm{~s}^{-1}$ as a perturbation of the case $B_{1}=0 \mathrm{~s}^{-1}$. The presence of the weaker sinusoidal input can, over one period, advance or delay the spike, depending on the relative phase of the two sinusoidal inputs at the time of the preceding spike. Over one period, the relative phase of the two sinusoids hardly changes because of the small difference in the frequencies of the sinusoidal inputs. The largest delay of the next spike due to the presence of the weaker input 1 takes place if the two sinusoids are 

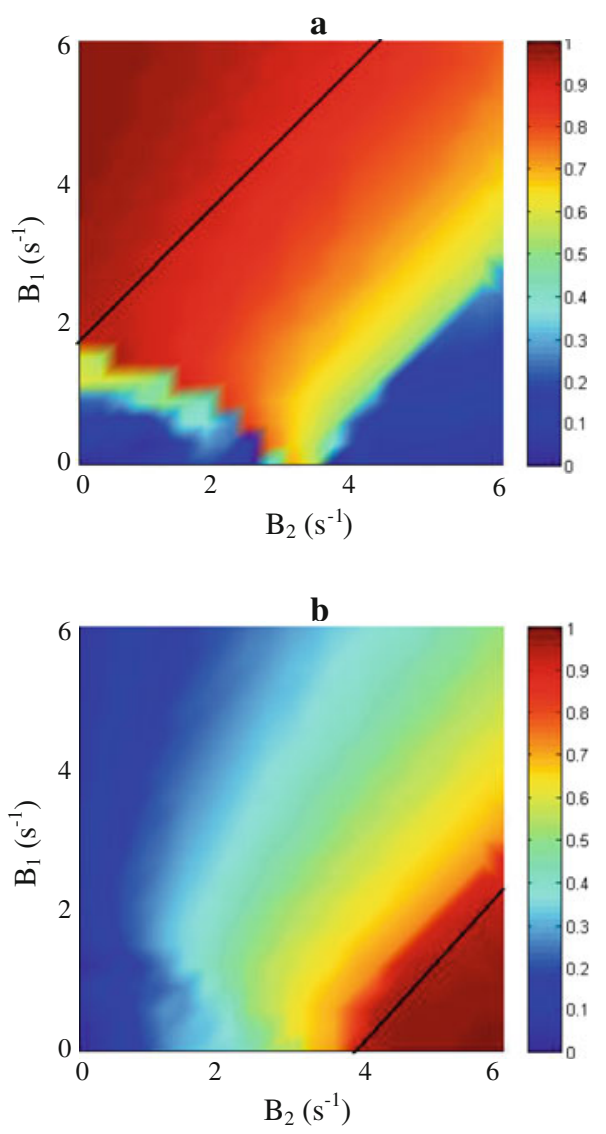

Fig. 3 Phase coherence to signal 1 (a) and to signal 2 (b) for the LIF neuron with membrane time constant $\tau=7 \mathrm{~ms}$. In a, the black line corresponds to the line $\left(B+B_{\mathrm{bif}}\left(\gamma_{1}\right), B\right)$, which gives the approximate boundary of the region of approximate locking to signal 1 with suppression of signal 2. In panel B the black line corresponds to the line $\left(B, B+B_{\text {bif }}\left(\gamma_{2}\right)\right)$, which gives the boundary of the region of approximate locking to signal 2 with suppression of signal 1

approximately in anti-phase. If for anti-phase of the two sinusoidal inputs $B_{2}-B_{1}>B_{\mathrm{bif}}\left(\gamma_{2}\right)$, then approximate locking occurs.

Figure 4a shows the evolution of the phase of spiking when, the relative phase of the two sinusoids changes. Firing of the LIF neuron shifts to earlier phases of the sinusoid with the larger amplitude ( $B_{2}$ in this case) when the relative phase of the sinusoidal inputs shifts from anti-phase to in-phase. The phase of spiking relative to input 2 is bounded below by the locking phase corresponding to the value $B_{2}-B_{1}$ and frequency $\gamma_{2}$. By a similar argument, we obtain an approximate upper bound for the locking phase corresponding to the input value $B_{2}+B_{1}$ and frequency $\gamma_{2}$. Since the decrease in frequency results in the decrease of $\theta$, this upper bound is just approximate and becomes less accurate as $B_{1}$ increases relative to $B_{2}$. Figure $4 \mathrm{~b}$ shows the variation of the phase of input 2 at the time of spiking of the LIF neuron. The black lines correspond to the bounds evaluated using the locking phases of $B_{2}-B_{1}$ and $B_{2}+B_{1}$. The LIF neuron tends to lock to
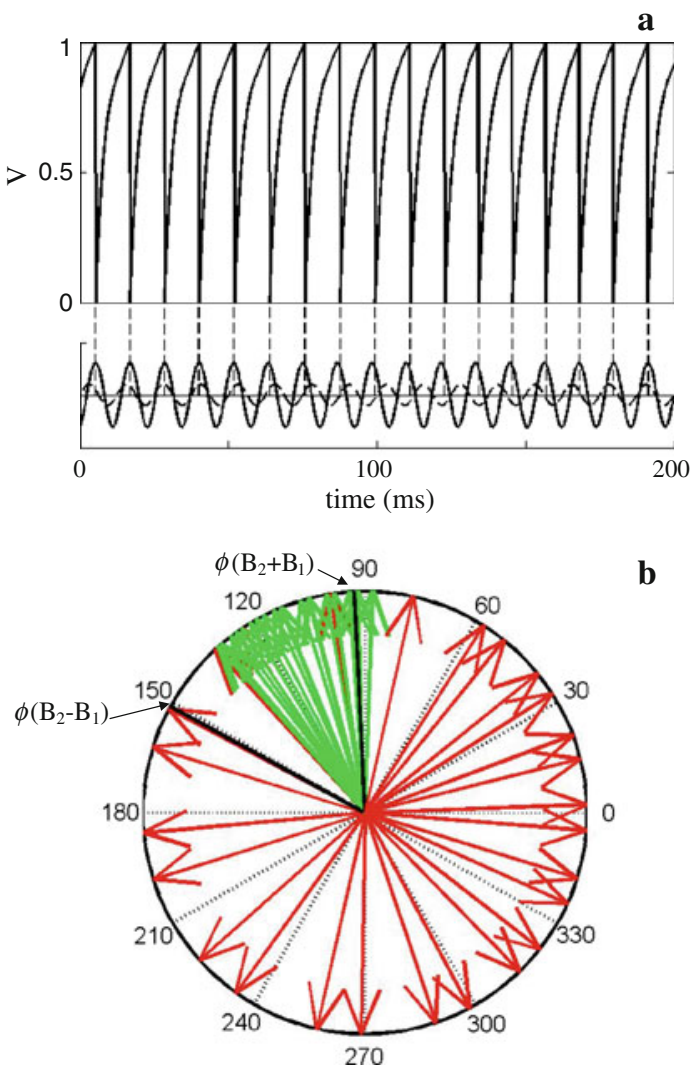

b

Fig. 4 Approximate locking of the LIF neuron to the stronger of two sinusoids for $\tau=7 \mathrm{~ms}, \gamma_{1}=40 \mathrm{~Hz}$, and $\gamma_{2}=43 \mathrm{~Hz}, B_{1}=2 \mathrm{~s}^{-1}$, $B_{2}=6.147 \mathrm{~s}^{-1}$. a The phase of the output approximately follows the phase of the stronger input (solid line), not that of the weaker sinusoid (dashed line). b Polar plot of the phases of the spikes relative to input 2 (green arrows) and relative to input 1 (red arrows). The phase of the sinusoid with the larger amplitude (sinusoid with solid line in a) is confined to a small region while the phase of the other input is homogeneously distributed around the circle. The black lines correspond to the locking phases for the input amplitudes $B_{2}-B_{1}$ and $B_{2}+B_{1}$, respectively, for frequency $\gamma_{2}$

sinusoidal input 2 (green arrows in Fig. 4b) at phases between the two bounds. Locking to sinusoidal input 1 (which has a much smaller amplitude) is absent and the spikes occur at more-or-less random phases (red arrows in Fig. 4b).

The black lines in Fig. 3a and $\mathrm{b}$ correspond to the bounds of approximate phase locking given by the above argument. Indeed, input 1 dominates at the upper/left of the black line in Fig. 3a and input 2 dominates at the lower/right of the black line in Fig. 3b. In both cases, the black lines give a good prediction of the boundary of approximate phase locking.

\subsection{The effect of varying $\gamma_{2}$ and $\tau$}

In Sect. 3.1 and 3.2, we showed that the regions for a large phase coherence to signal 1 and to signal 2 are roughly defined by the conditions: 


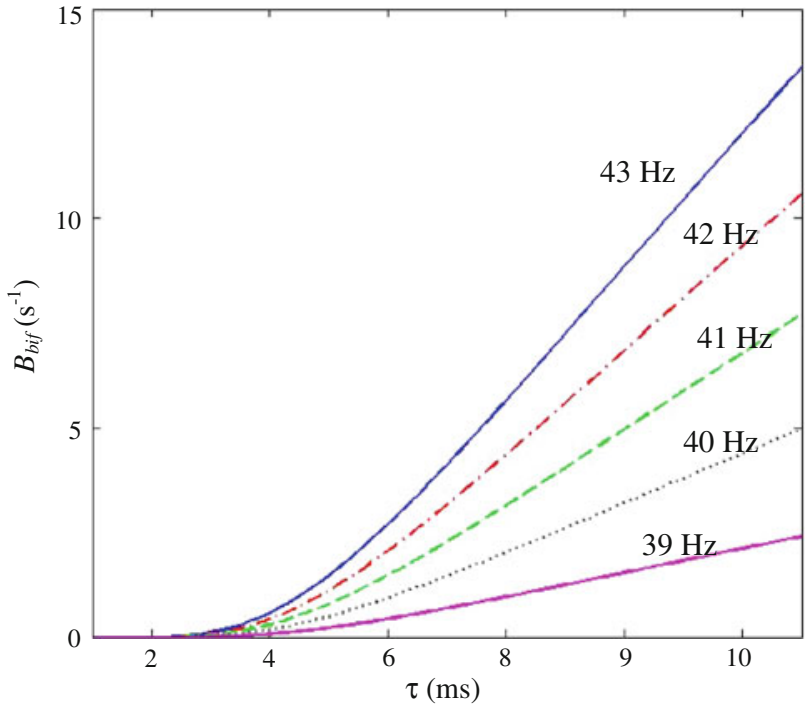

Fig. 5 The dependence of $B_{\text {bif }}$ on $\gamma$ and $\tau$ for the LIF neuron for various frequencies of the sinusoidal input

$B_{1}-B_{2}>B_{\text {bif }}\left(\gamma_{1}, \tau\right)$ and $B_{2}-B_{1}>B_{\text {bif }}\left(\gamma_{2}, \tau\right)$,

respectively. Therefore, the dependence of $B_{\mathrm{bif}}$ on $\gamma$ and $\tau$ is important for understanding the boundary regions of phase coherence for various values of $B_{\mathrm{bif}}, \gamma$, and $\tau$. Figure 5 shows the dependence of $B_{\text {bif }}$ on $\tau$ for various values of $\gamma$. Accord- ing to Eq. 5, $B_{\text {bif }}$ increases with $\gamma$ and $\tau$. For fixed $\gamma$, the increase in $\tau$ is linear for sufficiently large values of $\tau$, with the slope increasing as $\gamma$ increases.

Given the estimate of the approximate locking regions given by Eq. 7, we will study the effect of changes in the frequencies or the time constant on $B_{\text {bif }}$. Since $B_{\text {bif }}\left(\gamma_{2}, \tau\right)$ increases with $\gamma$, it follows in a straightforward way from Eq. 5 that larger amplitudes $B_{\text {bif }}$ are needed to achieve locking if the gamma frequency increases. Understanding the effect of changes in the time constant is more complicated: if $\tau$ decreases the leak becomes faster such that the firing frequency in the absence of gamma modulation decreases. In order to restore this base firing frequency to $38 \mathrm{~Hz}$, we must increase $\mu$. Given the adjustment of $\mu$ to maintain the base firing frequency at $38 \mathrm{~Hz}$, the prediction based on (5) is that gamma modulation becomes more effective for smaller values of $\tau$. This phenomenon is illustrated in Fig. 6, which shows the coherence between the output spikes of the LIF neuron and input 1 (left panels) and input 2 (right panels) for two different values of $\tau 7 \mathrm{~ms}$ (upper panels) and $13 \mathrm{~ms}$ (lower panels). The upper two panels are a replica of Fig. 3, showing regions of phase locking to both signal 1 and signal 2. In Fig. $6 \mathrm{c}$ and d, the time constant $\tau$ was changed from 7 to $13 \mathrm{~ms}$ and the value of $\mu$ was adjusted to achieve the same mean firing rate of 38 spikes/s without any sinusoidal input. The range of gamma modulation values $B_{1}$ and
Fig. 6 Coherence between the spike output of the LIF neuron and one of two sinusoidally modulated inputs ( 40 and $43 \mathrm{~Hz}$ ) for different values of $\tau$. a Phase coherence with signal $1(40 \mathrm{~Hz})$ for $\tau=7 \mathrm{~ms}$, b phase coherence with signal $2(43 \mathrm{~Hz})$ for $\tau=7 \mathrm{~ms}$, c phase coherence with signal $1(40 \mathrm{~Hz})$ for $\tau=13 \mathrm{~ms}$, $\mathbf{d}$ phase coherence with signal $2(43 \mathrm{~Hz})$ for $\tau=13 \mathrm{~ms}$
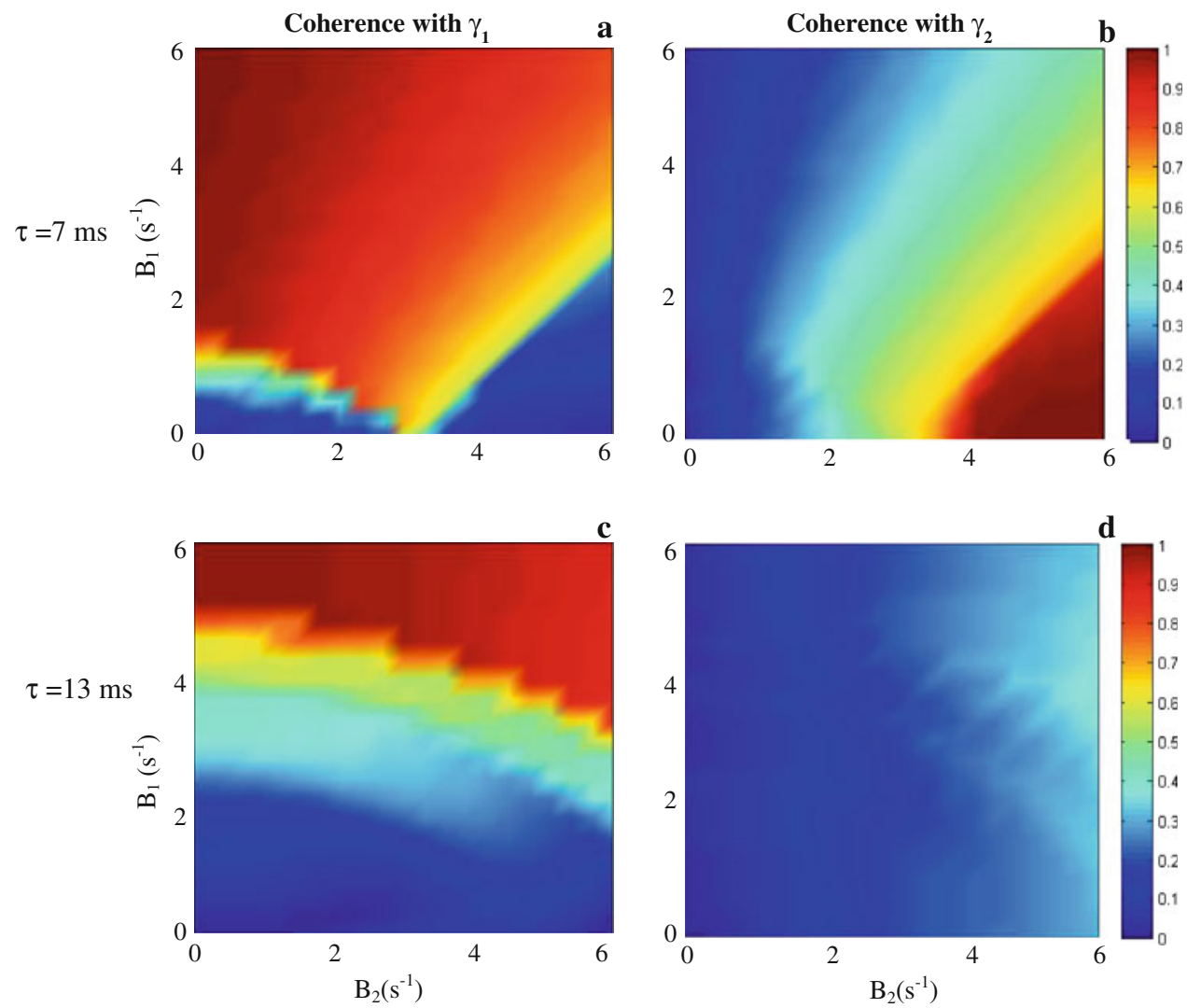
Fig. 7 Phase coherence between spike output of LIF neuron and two sinusoids with frequencies $\gamma_{1}=40 \mathrm{~Hz}$ and $\gamma_{2}=42 \mathrm{~Hz}$, rather than $\gamma_{2}=43 \mathrm{~Hz}$ as in the previous simulations. Panels $\mathbf{a}$ and $\mathbf{b}$ show phase coherence for $\tau=7 \mathrm{~ms}$, and panels $\mathbf{c}$ and $\mathbf{d}$ for $\tau=13 \mathrm{~ms}$. In all the pictures, the bounds for $B_{1}$ and $B_{2}$ are $\frac{3}{2} B_{\text {bif }}$. Note the similarity to the results obtained for $\gamma_{2}=43 \mathrm{~Hz}$ in Fig. 6a and b. This confirms the prediction that small changes in $\gamma_{1}$ and $\gamma_{2}$ do not significantly affect the phase coherence as long as the range of $B_{1}$ and $B_{2}$ is adjusted consistently (i.e. relative to $B_{\text {bif }}(\gamma, \tau)$ )
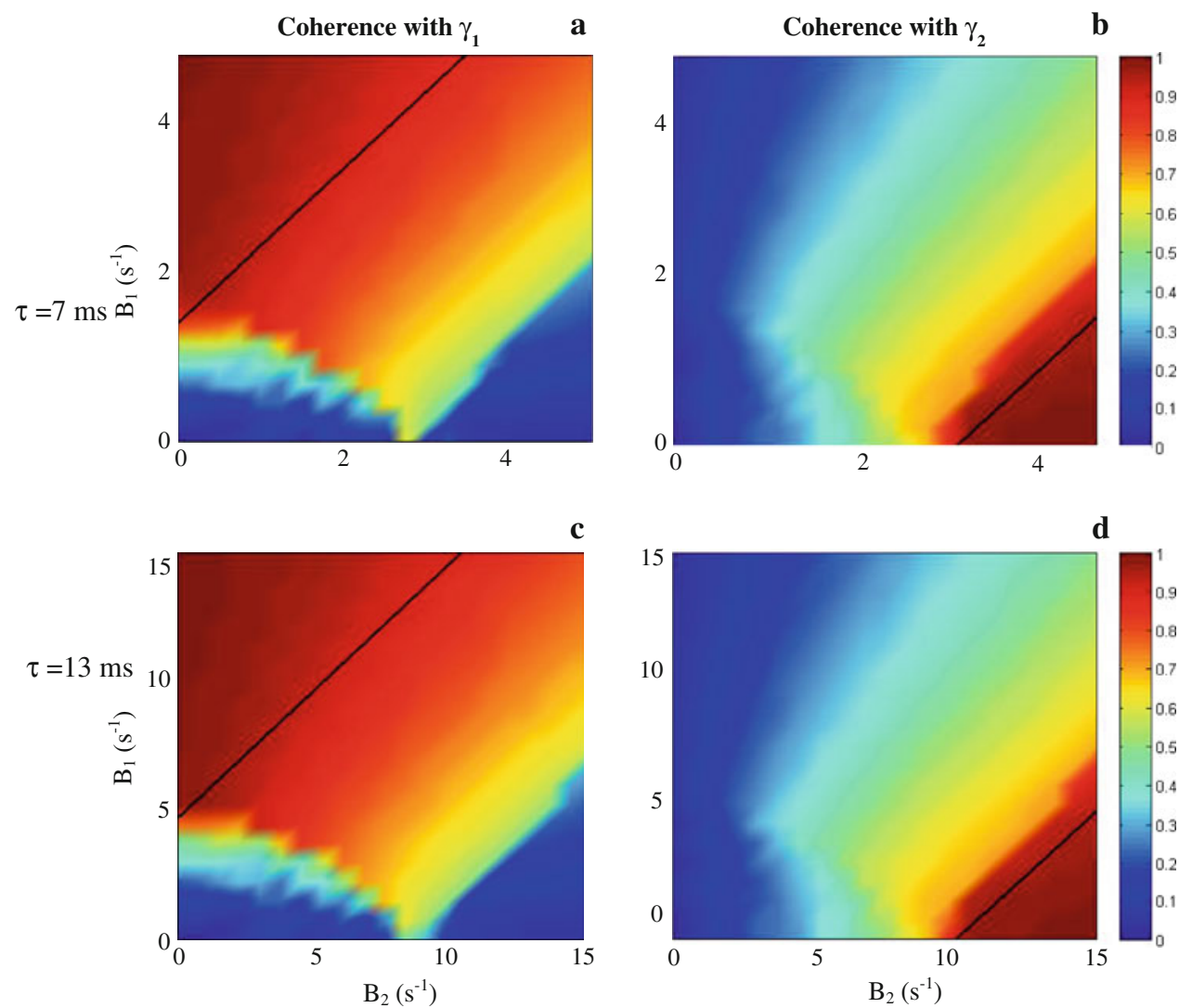

c

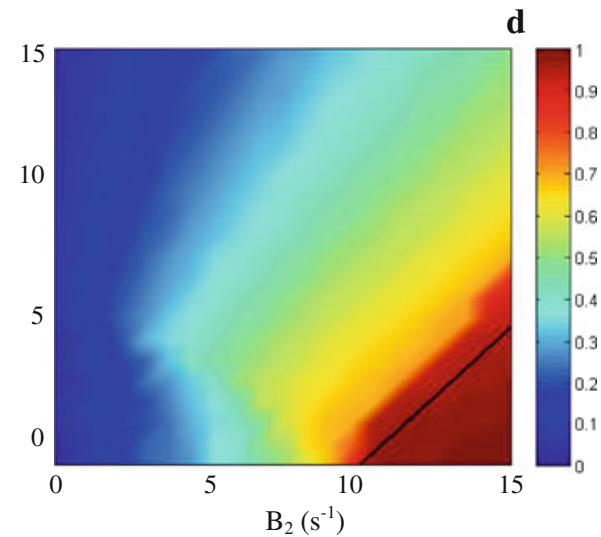

$B_{2}$ is the same in all panels of Fig. 6. For $\tau$ is $13 \mathrm{~ms}$, there is no longer coherence to signal 2 and almost no coherence to signal 1. This can be understood based on Fig. 5, which shows that the value of $B_{\text {bif }}\left(\gamma_{2}, \tau\right)$ approximately triples as $\tau$ changes from 7 to $13 \mathrm{~ms}$. Increasing the time constant to larger values changes the nature of the LIF neuron from that of a coincidence detector to an integrator (Kuhn et al., 2004).

The nice property of Eq. 7 is that it gives a prediction of the amplitudes $B_{1}$ and $B_{2}$ that provide approximate locking for a range of values for $\gamma_{1}, \gamma_{2}$ and $\tau$. For example, if we change the value of $\gamma_{2}$ and modify the range of $B_{1}$ and $B_{2}$ according to Eq. 6, the phase coherence plots should look very similar to the plots shown in Fig. 3. In order to illustrate this, we made simulations with $\gamma_{1}=40 \mathrm{~Hz}, \gamma_{2}=42 \mathrm{~Hz}$, and two different values of $\tau$, namely $\tau=7 \mathrm{~ms}$ and $\tau=13 \mathrm{~ms}$ (with an adjustment of $\mu$ as discussed in the preceding paragraph). The range of $B_{1}$ and $B_{2}$ was adjusted for the different time constants according to Eq. 6. The phase coherence plots are shown in Fig. 7. They are remarkably similar to the plots in Fig. 3.

In summary, the main finding of this section is that entrainment of the spike output of the LIF neuron by a sinusoidally modulated input in the gamma frequency range is possible. Entrainment is also possible in the case of two sinusoidal inputs. In that case the spike output is phase-locked to the stronger of the two gamma signals, provided that the difference between the amplitudes of the sinusoidally modu- lated signals exceeds the lower bound for entrainment (Eq. 7). Moreover, phase locking is easier when the time constant of the neuron is short.

\section{Other models}

In this section, we will briefly describe the results of computer simulations for the quadratic integrate and fire (QIF) neuron, the classical HH model and the PING model in response to two sinusoidally modulated inputs.

\subsection{Quadratic integrate and fire}

The quadratic integrate and fire neuron (QIF neuron) is described in detail in Latham et al. (2000). It is formally equivalent to the Theta neuron (Ermentrout and Kopell 1986) and has been often used in computational studies because it has analytical solutions for many input signals. In our study, we use the version used by (Börgers and Kopell 2005), given by

$\frac{\mathrm{d} V}{\mathrm{~d} t}=\frac{a}{\tau} V(V-1)+\frac{I}{C}$,

where $V$ is the membrane voltage (dimensionless), $C$ is the capacitance of the membrane and $I$ represents the external 
input to the neuron. $\tau$ is the time constant of the QIF neuron and $a$ is a parameter with the same dimension as $V$. In our study, we have taken $\frac{a}{\tau}=0.002 \mathrm{~s}^{-1}$ as in Börgers and Kopell (2005). When the voltage $V$ reaches the threshold value of $V=1$, its value is reset to 0 . There is a stable state and an unstable state for $I / C<0.5 \mathrm{~ms}^{-1}$, which merge for the input $I / C=0.5 \mathrm{~ms}^{-1}$ in a saddle-node bifurcation. For inputs $I / C>0.5 \mathrm{~ms}^{-1}$, the neuron spikes. In order to investigate the entrainment to external inputs, we have simulated the responses of a QIF neuron to an input consisting of two sinusoidally modulated signals, given by

$I / C=0.5+\mu+B_{1} \cos \left(2 \pi \gamma_{1} t\right)+B_{2} \cos \left(2 \pi \gamma_{2} t\right)$,

which is analogous to Eq. 2. The parameter $\mu>0 \mathrm{~s}^{-1}$ represents a constant input such that the mean firing rate of the QIF neuron is 38 spikes/s in the absence of the two gamma input signals. We have chosen the ranges of the amplitudes of the gamma signals to be sufficiently large so that locking occurs. Figure 8 shows the results of computer simulations. These results are very similar to the results in Fig. 3, obtained for the LIF neuron.

\subsection{Hodgkin-Huxley neuron}

In this section, we present the results of the simulations for the classical HH neuron, with the parameter values as given in Appendix 2. With these parameter settings, the HH neuron is a type-II neuron, which means that the neuron does not fire for small inputs and starts firing at a firing rate well above zero when the input exceeds a particular threshold. For the parameter values given in Appendix 2, this minimal firing frequency is approximately $50 \mathrm{~Hz}$. Hence, the frequency of the gamma inputs has been increased accordingly. In our simulations, we have set the value of $\mu$ so that the mean firing rate without any additional input (i.e. without the sinusoidally modulated input) of the $\mathrm{HH}$ neuron is $55 \mathrm{~Hz}$. The frequencies of the sinusoidally modulated gamma inputs were set to 57 and $60 \mathrm{~Hz}$, respectively.

Figure 9 shows the phase coherence between the spike output of the $\mathrm{HH}$ neuron and the $57 \mathrm{~Hz}$ sinusoidal input (a) and the $60 \mathrm{~Hz}$ sinusoidal input (b). The phase coherence depends on the amplitudes of the gamma-modulated signal (Fig. 9a, b) in a very similar way as for the LIF and the QIF neurons. The phase coherence is close to one if the amplitude
Fig. 8 Phase coherence between the spike output of QIF neuron and two sinusoidal input signals with frequencies 40 and $43 \mathrm{~Hz}$. a Phase coherence to signal 1 (frequency $40 \mathrm{~Hz}$ ), b phase coherence to signal 2 (frequency $43 \mathrm{~Hz}$ )
Fig. 9 Phase coherence between spike output of the classical HH neuron and two sinusoidal inputs as a function of the amplitude of the sinusoidal inputs. a Phase coherence of spike output with signal 1 (frequency $57 \mathrm{~Hz}$ ), b phase coherence with signal 2 (frequency $60 \mathrm{~Hz}$ ). The parameter values for the $\mathrm{HH}$ neuron are given in Appendix 1
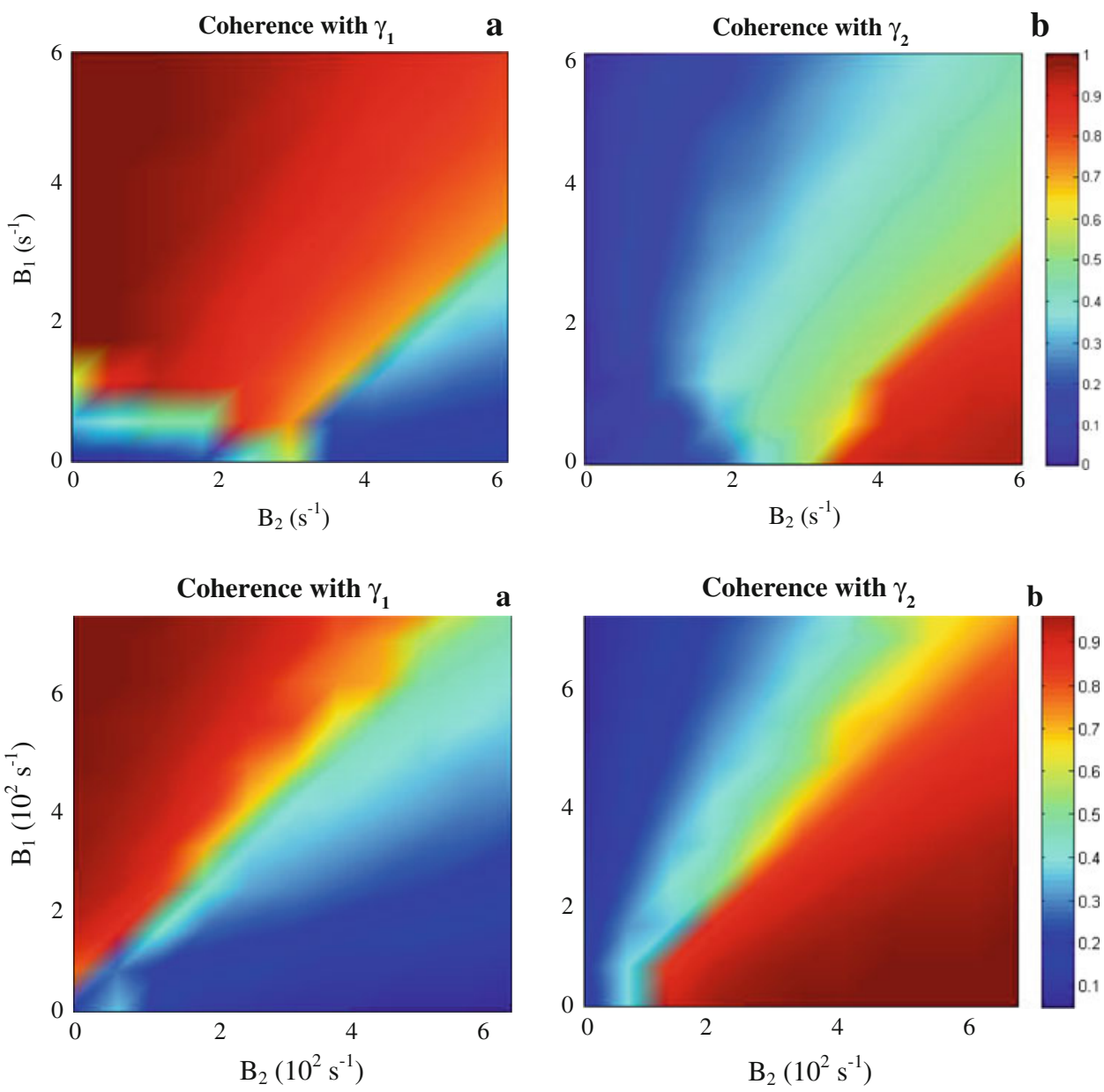
Fig. 10 Phase coherence between spike output of the excitatory neuron in the QIF-PING model and two sinusoidal input signals. a Phase coherence to signal 1 $(40 \mathrm{~Hz})$; $\mathbf{b}$ phase coherence to signal $2(43 \mathrm{~Hz})$
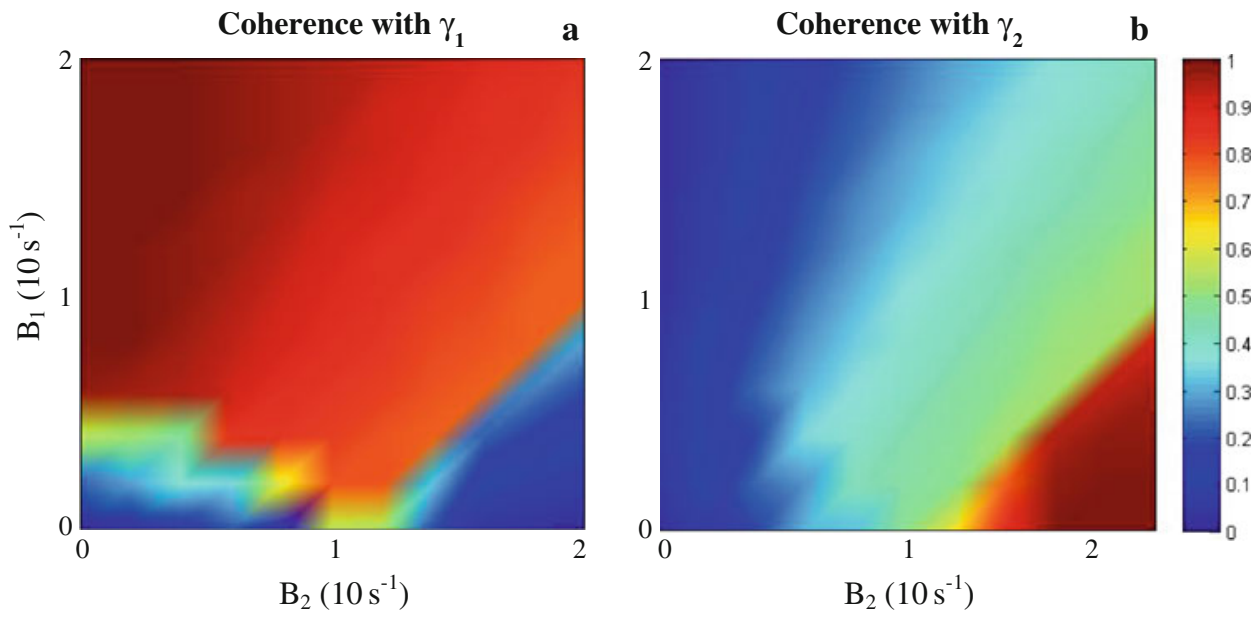

of one sinusoid exceeds the amplitude of the other sinusoid by about 2, qualitatively in agreement with Eq. 7 for the LIF neuron.

\subsection{PING model}

In the literature, a particular role has been attributed to the "PING" (Pyramidal-Interneuronal Network Gamma) mechanism (Whittington et al. 2000; Börgers and Kopell 2003, 2005 ) in the context of gamma rhythms, which arise from the interaction between excitatory pyramidal cells (E-cells) and inhibitory interneurons (I-cells). The underlying mechanism is that spikes of the I-cells inhibit and thereby synchronize the E-cells, and that spike volleys of the E-cells trigger synchronous spike volleys of the I-cells. Recently, Zeitler et al. (2008) have shown that a mechanism with interacting E- and I-cells may selectively respond to the stronger of two gamma-modulated signals. In order to compare the results obtained for the LIF, QIF and HH neurons, we have analysed a PING model with an excitatory QIF neuron, coupled to an inhibitory QIF neuron. Since theta neurons are equivalent to QIF neurons (Börgers and Kopell 2005), this PING model is equivalent to the PING model with theta neurons, proposed by Börgers and Kopell (2008). For brevity, we will refer to this model as the QIF-PING model.

The maximum synaptic coupling strength of the excitatory to the inhibitory neuron was $g_{\mathrm{EI}}=0.0510^{-3} \mathrm{~s}^{-1}$; that from the inhibitory to the excitatory neuron $g_{\mathrm{EI}}=0.210^{-3} \mathrm{~s}^{-1}$. The state of the synapse jumps to $1\left(s\left(t_{\mathrm{i}}\right)=1\right)$ at time $t_{i}$ of the spike and decays exponentially $\left(\frac{\mathrm{d} s}{\mathrm{~d} t}=-\frac{s}{\tau_{s}}\right)$. The time constant $\tau_{s}$ is $2 \mathrm{~ms}$ for the excitatory synapse and $10 \mathrm{~ms}$ for the inhibitory synapse.

Figure 10 shows the phase coherence between the spike output of the excitatory neuron with each of the two sinusoidal inputs for the QIF-PING model. In our simulation, we used a similar input as for the QIF neuron, see Eq. 8, with $\mu$ corresponding to the constant input needed for the QIF-PING model to fire with a firing rate of approximately
$38 \mathrm{~Hz}$. The phase-coherence plots, shown in Fig. 10a and b, are qualitatively very similar to that of the LIF neuron and QIF neuron. Quantitatively, the range of amplitudes is much smaller for the QIF neuron than for the PING model.

After the presentation of the results for the LIF, QIF and $\mathrm{HH}$ neurons and the PING model, the question arises to what extent the responses of the LIF, QIF and HH neurons on the one hand side and the PING model on the other hand differ from each other. First of all, the LIF and QIF neurons (see Figs. 7, 8) reveal entrainment for smaller gamma amplitudes than the PING model (Fig. 10). Moreover, large inputs give multiple spikes for the LIF and QIF neurons (see Fig. 2), which does not happen for the PING because of the strong inhibition of the excitatory cells by the inhibitory neurons after firing of the excitatory cells. Similarly, if two sinusoids with different frequencies, which provide input to the neuron, are in phase, they may elicit multiple spikes within a single cycle in the LIF and QIF neurons for sufficiently large amplitudes of the input. This would not happen for the PING model because of the inhibition after firing of the excitatory cell.

\section{Discussion}

The aim of the present study was to test the hypothesis that enhanced modulation of synchronized excitatory neuronal activity in the gamma frequency range provides an advantage over a less synchronized input for various types of neuron models using theoretical approaches and computer simulations. The work of Börgers and Kopell (2008) has already shown that more coherent excitatory stimuli may have a competitive advantage over less coherent ones for coupled excitatory and inhibitory neurons, in agreement with experimental observations. In their study, they investigated the responses of the PING model (consisting of an excitatory neuron and an inhibitory neuron, bi-directionally coupled to each other), to two input signals, each composed of a series of Gaussians constituting a periodic input in the gamma 
range. The main finding of Börgers and Kopell (2008) was that coherence matters more than amplitude of the input: the responses to the less coherent signal are suppressed by the more coherent signal as long as the inhibitory neuron provides sufficiently strong and long-lasting inhibition after a firing of the excitatory neuron. It is important to notice that this is due both to the timing of inhibition, as well as due to the leakiness of the neuron by inhibition.

In our study, we have taken a step back, trying to explain the basic mechanism of how entrainment by one of the inputs with suppression of the other can occur by studying the responses of single neurons. We have used the simplest possible choice of input consisting of a constant drive modulated by two sinusoids in the gamma range. The idea was that a larger gamma modulation corresponds to a more synchronized activity in the population of neurons that make up the gamma-modulated input, keeping the time-averaged input constant. The results of our study show that several types of neuron models, including the leaky integrate and fire neuron, the quadratic integrate and fire neuron and $\mathrm{HH}$ neuron model can respond selectively to one input in the presence of other inputs, as long as the difference in amplitudes of the two sinusoidal inputs is sufficiently large. The phase coherence between the spike output of the neuron and one of the inputs can be close to one if the difference in amplitudes of the gamma modulations exceeds a particular threshold. The output of the neuron does not reflect a linear summation of the contributions of both inputs; rather, it locks to the neuronal input with the larger gamma amplitude, even if the other input also has a significant amplitude. With these inputs, we obtain qualitatively similar results for single neurons and for E-I coupled neurons (PING model). The results for the PING model are in agreement with the results previously obtained by Börgers and Kopell (2008). The phase coherence between spike output and one of the sinusoidal inputs can be observed if the difference between the amplitudes of the sinusoidally modulated inputs is sufficiently large (see Eq. 7) and if the time constant of the neuron is relatively short (see Fig. 6). The latter agrees with the conclusion by Börgers and Kopell (2008) that not only timing of inhibition, but also leakiness is important for selective responses to the input signal with the largest gamma modulation.

In this study, we have used variations in gamma frequency amplitude as a measure for synchronized input. The basic idea was that the input to a neuron consists of a population of neurons. If these neurons fire in a coherent way, the population activity will reveal large variations, whereas the mean population activity will reveal small (noisy) fluctuations if all neuron fire completely uncorrelated. Therefore, variations in gamma frequency amplitude should be read as variations in the degree of input synchrony.

The issue of large gamma frequency amplitudes and the degree of synchrony in neuronal input is also relevant in the context of the leakiness of the neuron. Suppose that a LIF neuron fires at time $t=0$ and then receives a square input pulse of duration $\Delta t>0$ and strength $s>0$ with $w=s \Delta t$. We can think of $w$ as the 'total amount of charge injected'. This input will generate a spike if the membrane potential reaches the threshold, which is the case if and only if $w>\frac{\Delta t / \tau}{1-e^{-\Delta t / \tau}}$. This inequality shows that decreasing the leak time constant $\tau$ requires larger inputs. Therefore, if we compare two stimuli, one with a smaller $\Delta t$ (more 'coherent') and the other with a larger $\Delta t$ (less 'coherent'), the threshold (for $w$ ) is lower for the more coherent than for the less coherent one.

The phenomenon, that a neuron responds selectively to one out of several inputs, is highly important in the context of selective attention where subjects focus on one out of several features in the sensory environment. A good example is the simultaneous presentation of two stimuli within the receptive field of a neuron. Experimental studies in visual cortex (Treue and Maunsell 1996; Luck et al. 1997; Reynolds et al. 1999; Reynolds and Desimone 2003) have demonstrated that the output of a neuron may be related to either input, depending on whether attention is directed to one or the other stimulus. This bias in favour of the attended stimulus is correlated with enhanced gamma band $(30-80 \mathrm{~Hz})$ synchronization (Fries et al. 2001; Gruber et al. 1999; Taylor et al. 2005). Zeitler et al. (2008) showed that a relatively simple feed-forward model with excitatory and inhibitory neurons could explain these results by assuming that the relevant stimulus is encoded by a larger amplitude of the gamma-rhythm than the nonpreferred stimulus. The results of our study show that selective responses to multiple inputs can also be observed for single neurons and for pairs of neurons coupled by excitatory and inhibitory synaptic connections.

Börgers and Kopell (2008) argued that suppression of the less-coherent inputs is due both to the timing of inhibition as well as to the effect that inhibition affects the leakiness of the neuron. A similar mechanism explains the results in our study for each of the neurons that we considered (i.e. the LIF, QIF and $\mathrm{HH}$ models). Regarding the timing of inhibition in our study, there are several aspects that matter; first of all, it takes some time to generate a new action potential when the neuron has just fired. Therefore, if a strong stimulus causes the neuron to fire, it takes some time before the neuron can generate another action potential due to other inputs. Moreover, the spike occurs in the phase when the stronger sinusoid is positive, but by the time that the membrane potential returns to the threshold level the sinusoid corresponding to the stronger stimulus reaches its negative phase, so that the total input may be too small to cause an action potential. Notice that we carefully limited the amplitude of our sinusoidal inputs such that the total input was always positive, even at the through of the sinusoid, and that the neurons in our study never received inhibition. Larger amplitudes of the sinusoids, giving rise 
to inhibition, would have enhanced the effect of timing of inhibition, strengthening the enhanced responses to the signal with the large gamma amplitude. Therefore, timing of inhibition partly explains why the neuron is less sensitive to other uncorrelated input signals. A second important explanation for selective responses to signals with larger gamma amplitudes comes from the leak-time constant of the neuron (see Eqs. 4, 5 and 7; Fig. 6). Leakiness promotes enhanced responses to the sinusoid with the larger gamma amplitude (see Fig. 7), in agreement with previous results for the PING model (Börgers and Kopell 2008).

Since the inputs in our study were sinusoids, the question arises whether the patterns of selective entrainment we have found persist for noisy inputs, such as band pass filtered white noise, Poisson spike input or experimental data as measured by MEG and local field potentials. Our results show that the PING model requires larger amplitudes of the gamma signal for entrainment, which makes it less vulnerable for noise than the LIF, QIF and HH models. We hypothesize that the presence of inhibition in the PING model will be instrumental for the persistence of entrainment, in agreement with previous results by Börgers and Kopell $(2003,2005)$.

The finding of prominent gamma-related activity in neuronal signals has led to several hypotheses regarding its functional role, in particular on the role of coherent activity in the gamma-frequency range within a population of neurons. It has been hypothesized that a coherent input may (i) enhance the effectiveness of excitatory signals (Murthy and Fetz 1994; Singer 1999), (ii) may weaken the effectiveness of inhibitory signals (Börgers et al. 2005; Börgers and Kopell 2005; Lumer 2000; Tiesinga 2002; Tiesinga et al. 2004), (iii) may create modulations of excitability, thereby providing windows for effective communication (Fries 2005), (iv) may play an important role in the creation of cell assemblies (Hayon et al. 2005) or (v) may serve as a label for solving the 'binding problem' (Engel et al. 2001; Singer and Gray 1995; von der Malsburg and Schneider 1986). Although these hypothesis are clearly different, they may not be exclusive. The results of this study provide strong evidence for the first hypothesis that gamma-encoding may enhance the effectiveness of an excitatory neuronal signal and may suppress or annihilate neuronal responses to inputs with less prominent gamma encoding.

Based on our results, we hypothesize that gammaoscillations in itself may not carry specific sensory information, but rather that gamma modulations operate as a vehicle to encode sensory information such that this information is processed accurately and efficiently by subsequent groups of neurons. This is in agreement with the CTC hypothesis proposed by Fries (2005); Fries et al. (2007) and the possible role of the PING mechanism (Börgers and Kopell 2008). The CTC hypothesis emphasizes the role of the membrane time constant. By balanced excitation and inhibition, the average subthreshold membrane potential does not change, but the resistance of the cell membrane decreases, and this decreases the time constant of the cell membrane. With the shorter time constant, the neuron behaves more like a coincidence detector and tends to respond better to synchronized input (Azouz and Gray 2000; Kuhn et al. 2004), thereby greatly amplifying the advantage of a more coherent excitatory input $A$ over a less coherent competitor $B$. Regarding the PING mechanism (Börgers and Kopell 2003, 2005; Whittington et al. 2000), a synchronized gamma frequency train $A$ of excitatory input pulses triggers synchronous spike volleys of the I-cells, which then synchronize the E-cells by inhibitory spike volleys. The timing of the inhibition from the inhibitory neurons after the spike volleys of a coherent stimulus A to the excitatory neurons decreases the contribution of a competing, less coherent pulse train $B$ that is uncorrelated with the more coherent stimulus A (Börgers and Kopell 2008). The notion of modulation of excitability, which is the key in the CTC hypothesis by Fries (2005), also implies that oscillatory activity is a mechanism for efficient information transfer, rather than a carrier of stimulus-specific information.

The CTC hypothesis postulates that oscillatory activity is processed accurately if the periodic excitatory input arrives at the neuron near the peak of the oscillatory excitability. However, the CTC hypothesis does not explain how the proper (optimal) phase relation between oscillatory input and oscillatory excitability is achieved. Our study shows that a sinusoidal input gives rise to a small range of stable phase relations in the range between approximately $90^{\circ}$ to $135^{\circ}$. This implies that the spike in the output neuron is generated at the peak of the input sinusoid or slightly later. This implies that the stable phase for entrainment is such that the sinusoidal input arrives at or just prior to the peak excitability of the neuron. Therefore, our study shows that the intrinsic dynamics of neurons and neuronal coupling ensures that the modulations of excitability of the neuron adjust to a phase relative to the periodic input such, that the excitability is near its maximum at the peak input.

Acknowledgements This work was supported by the Netherlands Organization for Scientific Research (NWO) grant 635.100.019 to SG. We also acknowledge very useful and stimulating discussions with Christoph Börgers.

Open Access This article is distributed under the terms of the Creative Commons Attribution Noncommercial License which permits any noncommercial use, distribution, and reproduction in any medium, provided the original author(s) and source are credited.

\section{Appendix}

\section{A. Dynamics of membrane potential}

In this section, we will derive an explicit solution of the equation for the leaky integrate and fire neuron with a sinusoidal input with amplitude B and frequency $\gamma$ : 
$\frac{\mathrm{d} V}{\mathrm{~d} t}=-\frac{1}{\tau}\left(V-V_{0}\right)+\mu+B \sin (2 \pi \gamma t)$.

Using the integrating factor $e^{t / \tau}$

$$
\left(\frac{\mathrm{d} V}{\mathrm{~d} t}+\frac{1}{\tau} V\right) e^{\frac{t}{\tau}}=\left(\frac{1}{\tau} V_{0}+\mu\right) e^{\frac{t}{\tau}}+B \sin (2 \pi \gamma t) e^{\frac{t}{\tau}}
$$

the differential equation becomes:

$$
\frac{\mathrm{d}}{\mathrm{d} t}\left(V e^{\frac{t}{\tau}}\right)=\left(\frac{1}{\tau} V_{0}+\mu\right) e^{\frac{t}{\tau}}+B \sin (2 \pi \gamma t) e^{\frac{t}{\tau}} .
$$

Integration over the time interval $t$ from $t_{0}$ to $t+t_{0}$ gives

$$
\begin{aligned}
V\left(t+t_{0}\right) e^{\frac{t+t_{0}}{\tau}}= & \int_{t_{0}}^{t+t_{0}}\left(\frac{1}{\tau} V_{0}+\mu\right) e^{\frac{s}{\tau}} \mathrm{d} s \\
& +B \int_{t_{0}}^{t+t_{0}} \sin (2 \pi \gamma t) e^{\frac{s}{\tau}} \mathrm{d} s .
\end{aligned}
$$

Now, we evaluate the two integrals separately.

$$
\int_{t_{0}}^{t+t_{0}}\left(\frac{1}{\tau} V_{0}+\mu\right) e^{\frac{s}{\tau}} \mathrm{d} s=\left(V_{0}+\tau \mu\right)\left(e^{\frac{t+t_{0}}{\tau}}-e^{\frac{t_{0}}{\tau}}\right) .
$$

The other integral can be evaluated using integration by parts (twice)

$$
\begin{aligned}
& \int_{t_{0}}^{t+t_{0}} \sin (2 \pi \gamma t) e^{\frac{s}{\tau}} \mathrm{d} s=\left[\tau \sin (2 \pi \gamma s) e^{\frac{s}{\tau}}\right]_{t_{0}}^{t+t_{0}} \\
& -2 \pi \gamma \tau \int_{t_{0}}^{t+t_{0}} \cos (2 \pi \gamma t) e^{\frac{s}{\tau}} \mathrm{d} s \\
& =\tau\left[\sin \left(2 \pi \gamma\left(t+t_{0}\right)\right) e^{\frac{t+t_{0}}{\tau}}-\sin \left(2 \pi \gamma t_{0}\right) e^{\frac{t_{0}}{\tau}}\right] \\
& -2 \pi \gamma \tau^{2}\left[\cos \left(2 \pi \gamma\left(t+t_{0}\right)\right) e^{\frac{t+t_{0}}{\tau}}-\cos \left(2 \pi \gamma t_{0}\right) e^{\frac{t_{0}}{\tau}}\right] \\
& -4 \pi^{2} \gamma^{2} \tau^{2} \int_{t_{0}}^{t+t_{0}} \sin (2 \pi \gamma t) e^{\frac{s}{\tau}} \mathrm{d} s .
\end{aligned}
$$

Transferring the term $-4 \pi^{2} \gamma^{2} \tau^{2} \int_{t_{0}}^{t+t_{0}} \sin (2 \pi \gamma t) e^{\frac{s}{\tau}} \mathrm{d} s$ to the other side gives

$$
\begin{aligned}
(1+ & \left.4 \pi^{2} \gamma^{2} \tau^{2}\right) \int_{t_{0}}^{t+t_{0}} \sin (2 \pi \gamma t) e^{\frac{s}{\tau}} \mathrm{d} s \\
= & \tau\left[\sin \left(2 \pi \gamma\left(t+t_{0}\right)\right) e^{\frac{t+t_{0}}{\tau}}-\sin \left(2 \pi \gamma t_{0}\right) e^{\frac{t_{0}}{\tau}}\right] \\
& -2 \pi \gamma \tau^{2}\left[\cos \left(2 \pi \gamma\left(t+t_{0}\right)\right) e^{\frac{t+t_{0}}{\tau}}-\cos \left(2 \pi \gamma t_{0}\right) e^{\frac{t_{0}}{\tau}}\right] .
\end{aligned}
$$

Now we divide both sides by $\left(1+4 \pi^{2} \gamma^{2} \tau^{2}\right)$ :

$$
\begin{aligned}
\int_{t_{0}}^{t+t_{0}} \sin (2 \pi \gamma t) e^{\frac{s}{\tau}} \mathrm{d} s=\frac{\tau}{1+4 \pi^{2} \gamma^{2} \tau^{2}} \\
\times\left[\sin \left(2 \pi \gamma\left(t+t_{0}\right)\right) e^{\frac{t+t_{0}}{\tau}}-\sin \left(2 \pi \gamma t_{0}\right) e^{\frac{t_{0}}{\tau}}\right] \\
-\frac{2 \pi \gamma \tau^{2}}{1+4 \pi^{2} \gamma^{2} \tau^{2}} \\
\times\left[\cos \left(2 \pi \gamma\left(t+t_{0}\right)\right) e^{\frac{t+t_{0}}{\tau}}-\cos \left(2 \pi \gamma t_{0}\right) e^{\frac{t_{0}}{\tau}}\right] \\
=\frac{\sqrt{1+4 \pi^{2} \gamma^{2} \tau^{2}}}{\sqrt{1+4 \pi^{2} \gamma^{2} \tau^{2}}} \\
\times\left[\sin \left(2 \pi \gamma\left(t+t_{0}\right)\right) e^{\frac{t+t_{0}}{\tau}}-\sin \left(2 \pi \gamma t_{0}\right) e^{\frac{t_{0}}{\tau}}\right] \\
- \\
\quad \frac{2 \pi \gamma \tau}{\sqrt{1+4 \pi^{2} \gamma^{2} \tau^{2}}} \\
\left.\times\left[\cos \left(2 \pi \gamma\left(t+t_{0}\right)\right) e^{\frac{t+t_{0}}{\tau}}-\cos \left(2 \pi \gamma t_{0}\right) e^{\frac{t_{0}}{\tau}}\right]\right) .
\end{aligned}
$$

We now define $\theta=\arctan (2 \pi \gamma \tau)$ and use the formula $\sin (\alpha-\beta)=\sin (\alpha) \cos (\beta)-\sin (\beta) \cos (\alpha)$ to obtain

$$
\begin{aligned}
& \int_{t_{0}}^{t+t_{0}} \sin (2 \pi \gamma t) e^{\frac{s}{\tau}} \mathrm{d} s=\frac{\tau}{\sqrt{1+4 \pi^{2} \gamma^{2} \tau^{2}}} \\
& \quad \times\left(\sin \left(2 \pi \gamma\left(t+t_{0}\right)-\theta\right) e^{\frac{t+t_{0}}{\tau}}-\sin \left(2 \pi \gamma t_{0}-\theta\right) e^{\frac{t_{0}}{\tau}}\right) .
\end{aligned}
$$

Combining all these results, we obtain the explicit expression

$$
\begin{aligned}
V\left(t+t_{0}\right)= & \left(\tau \mu+V_{0}\right)\left(1-e^{-\frac{t}{\tau}}\right) \\
& -B \frac{\tau}{\sqrt{4 \pi^{2} \gamma^{2} \tau^{2}+1}} \sin \left(2 \pi \gamma t_{0}-\theta\right) e^{-\frac{t}{\tau}} \\
& +B \frac{\tau}{\sqrt{4 \pi^{2} \gamma^{2} \tau^{2}+1}} \sin \left(2 \pi \gamma t_{0}-\theta+2 \pi \gamma t\right)
\end{aligned}
$$

B. Computation of the fixed point

In Eq. A1, we insert $t=1 / \gamma$, define $\varphi=2 \pi \gamma t_{0}$ and set the LHS to $V_{\theta}$. This gives:

$$
\begin{aligned}
V_{\theta}= & \left(\tau \mu+V_{0}\right)\left(1-e^{-\frac{1}{\tau \gamma}}\right) \\
& +B \frac{\tau}{\sqrt{4 \pi^{2} \gamma^{2} \tau^{2}+1}} \sin (\varphi-\theta)\left(1-e^{-\frac{1}{\tau \gamma}}\right)
\end{aligned}
$$

We define $\mu_{\gamma}$ to be the amount of constant input needed for spiking with frequency $\gamma$. Then, since $V_{\theta}=\left(\tau \mu_{\gamma}+V_{0}\right)$ $\left(1-e^{-\frac{1}{\tau \gamma}}\right)$, we obtain:

$\tau \mu_{\gamma}-\tau \mu=B \frac{\tau}{\sqrt{4 \pi^{2} \gamma^{2} \tau^{2}+1}} \sin (\varphi-\theta)$. 
Dividing both sides by $\frac{\tau}{\sqrt{4 \pi^{2} \gamma^{2} \tau^{2}+1}}$, we obtain formula (4).

Recall (see Sect. 3.1) that as $B$ becomes too large, this formula does not define a 1-to-1 phase-locked solution due to the appearance of a spike at an earlier time (see Fig. 2).

\section{Computation of stability}

For a given initial phase $\varphi=2 \pi \gamma t_{0}$ at the moment of a spike (in other words, the LIF neuron fires at $t=t_{0}$ ), we can write an implicit condition for the time it takes until the next spike. This is done using Eq. A1 with the LHS replaced with $V_{\theta}$ and $t$ replaced with $T(\varphi)$. This yields the following expression

$$
\begin{aligned}
V_{\theta}= & \left(\tau \mu+V_{0}\right)\left(1-e^{-\frac{T(\varphi)}{\tau}}\right) \\
& -B \frac{\tau}{\sqrt{4 \pi^{2} \gamma^{2} \tau^{2}+1}} \sin (\varphi-\theta) e^{-\frac{T(\varphi)}{\tau}} \\
& +B \frac{\tau}{\sqrt{4 \pi^{2} \gamma^{2} \tau^{2}+1}} \sin (\varphi-\theta+2 \pi \gamma T(\varphi))
\end{aligned}
$$

which defines $T(\varphi)$ implicitly. Recall the return map $\Pi(\varphi)=$ $\varphi+2 \pi \gamma T(\varphi)(\bmod 2 \pi)$. Recall also that if $B>B_{\text {bif }}$, where $B_{\text {bif }}$ is defined by (5) then this map has two fixed points, one satisfying $\theta<\varphi<\theta+\pi / 2$ and the other satisfying $\pi / 2+\theta<\varphi<\theta+\pi$. Here, we focus on the first type of fixed point and denote it $\varphi_{l}$.

Recall that $\varphi_{l}$ exists for $B$ sufficiently small. We will show that $0<\frac{\mathrm{d} \Pi}{\mathrm{d} \varphi}\left(\varphi_{l}\right)<1$ which implies that $\varphi_{l}$ is always stable (only a weaker estimate $-1<\frac{\mathrm{d} \Pi}{\mathrm{d} \varphi}\left(\varphi_{l}\right)<1$ is needed). The other fixed point can be shown to be unstable by a similar argument.

Differentiating both sides of (A2) with respect to $\varphi$ we obtain a formula for $\mathrm{d} T / \mathrm{d} \varphi$ :

$$
\begin{aligned}
0= & \left(\frac{\left(\tau \mu+V_{0}\right)}{\tau}+B \frac{1}{\sqrt{4 \pi^{2} \gamma^{2} \tau^{2}+1}} \sin (\varphi-\theta)\right) e^{-\frac{T(\varphi)}{\tau}} \\
& \times \frac{\mathrm{d} T(\varphi)}{\mathrm{d} \varphi}+2 \pi \gamma B \frac{\tau}{\sqrt{4 \pi^{2} \gamma^{2} \tau^{2}+1}} \\
& \times \cos (\varphi-\theta+2 \pi \gamma T(\varphi)) \\
& \times \frac{\mathrm{d} T(\varphi)}{\mathrm{d} \varphi}-B \frac{\tau}{\sqrt{4 \pi^{2} \gamma^{2} \tau^{2}+1}} \cos (\varphi-\theta) e^{-\frac{T(\varphi)}{\tau}} \\
& +B \frac{\tau}{\sqrt{4 \pi^{2} \gamma^{2} \tau^{2}+1}} \cos (\varphi-\theta+2 \pi \gamma T(\varphi)) .
\end{aligned}
$$

Now if we let $\varphi=\varphi_{l}$ (the phase corresponding to locking) then the above expression simplifies since $T\left(\varphi_{l}\right)=1 / \gamma$ and Eq. 4 holds. We include one intermediate level of the computation:

$$
\begin{aligned}
0= & \left(\frac{\left(\tau \mu+V_{0}\right)}{\tau}+\left(\mu_{\gamma}-\mu\right)\right) e^{-\frac{1}{\tau \gamma}} \frac{\mathrm{d} T}{\mathrm{~d} \varphi}\left(\varphi_{l}\right) \\
& +2 \pi \gamma \tau\left(\mu_{\gamma}-\mu\right) \cot \left(\varphi_{l}-\theta\right) \frac{\mathrm{d} T}{\mathrm{~d} \varphi}\left(\varphi_{l}\right) \\
& -\cot \left(\varphi_{l}-\theta\right) \tau\left(\mu_{\gamma}-\mu\right)\left(1-e^{-\frac{1}{\tau \gamma}}\right) .
\end{aligned}
$$

Finally, we have

$$
\begin{aligned}
& \frac{\mathrm{d} T}{\mathrm{~d} \varphi}\left(\varphi_{l}\right)= \\
& -\frac{\cot \left(\varphi_{l}-\theta\right) \tau\left(\mu_{\gamma}-\mu\right)\left(1-e^{-\frac{1}{\tau \gamma}}\right)}{\left(\frac{\left(\tau \mu+V_{0}\right)}{\tau}+\left(\mu_{\gamma}-\mu\right)\right) e^{-\frac{1}{\tau \gamma}}+2 \pi \gamma \tau\left(\mu_{\gamma}-\mu\right) \cot \left(\varphi_{l}-\theta\right)} .
\end{aligned}
$$

It follows that $\frac{\mathrm{d} T}{\mathrm{~d} \varphi}\left(\varphi_{l}\right)=0$ for $\varphi_{l}=\theta+\pi / 2$ (the bifurcation value). When $\varphi_{l}<\theta+\pi / 2$ (which is the case except at the bifurcation) then $\frac{\mathrm{d} T}{\mathrm{~d} \varphi}\left(\varphi_{l}\right)<0$. Moreover, $\frac{\mathrm{d} T}{\mathrm{~d} \varphi}\left(\varphi_{l}\right)>-\frac{1}{2 \pi \gamma}$; this follows from the estimate

$$
\begin{aligned}
& \frac{\cot \left(\varphi_{l}-\theta\right) \tau\left(\mu_{\gamma}-\mu\right)\left(1-e^{-\frac{1}{\tau \gamma}}\right)}{\left(\frac{\left(\tau \mu+V_{0}\right)}{\tau}+\left(\mu_{\gamma}-\mu\right)\right) e^{-\frac{1}{\tau \gamma}}+2 \pi \gamma \tau\left(\mu_{\gamma}-\mu\right) \cot \left(\varphi_{l}-\theta\right)} \\
& \leq \frac{\cot \left(\varphi_{l}-\theta\right) \tau\left(\mu_{\gamma}-\mu\right)\left(1-e^{-\frac{1}{\tau \gamma}}\right)}{2 \pi \gamma \tau\left(\mu_{\gamma}-\mu\right) \cot \left(\varphi_{l}-\theta\right)}=\frac{1-e^{-\frac{1}{\tau \gamma}}}{2 \pi \gamma} \\
& <\frac{1}{2 \pi \gamma} .
\end{aligned}
$$

Since $\Pi(\varphi)=\varphi+2 \pi \gamma T(\varphi)$, it follows that for any $\varphi_{l}$ as specified at the beginning of this section the estimate $0<$ $\frac{d \Pi}{d \varphi}\left(\varphi_{l}\right)<1$ holds.

\section{Appendix 2}

The parameters for the Hodgkin-Huxley neuron

This appendix gives the parameter values for the $\mathrm{HH}$ neuron that have been used in this study. The parameters for the $\mathrm{HH}$ neuron are the following. The reversal potentials for sodium, potassium and the leak channel are set to $E_{\mathrm{Na}}=45 \mathrm{mV}, E_{\mathrm{K}}=-82 \mathrm{mV}$ and $E_{\mathrm{L}}=$ $-59.387 \mathrm{mV}$, respectively. The maximum conductances are $g_{\mathrm{Na}}=120\left(\mathrm{mS} / \mathrm{cm}^{2}\right), g_{\mathrm{K}}=36\left(\mathrm{mS} / \mathrm{cm}^{2}\right)$ and $g_{\mathrm{L}}=$ $0.3\left(\mathrm{mS} / \mathrm{cm}^{2}\right)$. The rate constants are given by

$$
\begin{aligned}
\alpha_{m} & =\frac{(V+45) / 10}{1-\exp (-(V+45) / 10)} \\
\alpha_{h} & =0.07 \exp (-(V+70) / 20) \\
\alpha_{n} & =\frac{(V+60) / 100}{1-\exp (-(V+60) / 100)} \\
\beta_{m} & =4^{*} \exp (-(V+70) / 18)
\end{aligned}
$$




$$
\begin{aligned}
& \beta_{h}=\frac{1}{1-\exp (-(V+40) / 10)} \\
& \beta_{n}=0.125^{*} \exp (-(V+70) / 80)
\end{aligned}
$$

\section{References}

Adrian ED (1932) The mechanisms of nervous action: electrical studies of the neurone. University of Pennsylvania Press, Philadelphia

Averbeck B, Latham PE, Pouget A (2006) Neural correlations, population coding and computation. Nat Rev Neurosci 7:358-366

Azouz R, Gray CM (2000) Dynamic spike threshold reveals a mechanism for synaptic coincidence detection in cortical neurons in vivo. Proc Natl Acad Sci USA 97(14):8010-8015

Börgers C, Kopell N (2003) Synchronization in networks of excitatory and inhibitory neurons with sparse, random connectivity. Neural Comput 15(3):509-539

Börgers C, Kopell N (2005) Effects of noisy drive on rhythms in networks of excitatory and inhibitory neurons. Neural Comput 17(3):557-608

Börgers C, Kopell NJ (2008) Gamma oscillations and stimulus selection. Neural Comput 20(2):383-414

Börgers C, Epstein S, Kopell N (2005) Background gamma rhythmicity and attention in cortical local circuits: a computational study. Proc Natl Acad Sci USA 102(19):7002-7007

Börgers C, Krupa M, Gielen S (2010) The response of classical Hodgkin-Huxley neurons to an inhibitory input pulse. J Comput Neurosci doi:10.1007/s10827-010-0233-8

Dayan P, Abbott LF (2001) Theoretical neuroscience. Computational and mathematical modelling of neural systems. The MIT Press, Cambridge

Engel AK, Fries P, Singer W (2001) Dynamic predictions: oscillations and synchrony in top-down processing. Nat Rev Neurosci 2: 704-716

Ermentrout GB, Kopell N (1986) Parabolic bursting in an excitable system coupled with a slow oscillation. SIAM J Appl Math 46: 233-253

Escalona J, Jorge V, Jose JV, Tiesinga P (2002) Entrainment, Arnold tongues, and duality in a periodically driven integrate-and-fire model. Neurocomputing 44-46:91-96

Fries P (2005) A mechanism for cognitive dynamics: neuronal communication through neuronal coherence. Trends Cogn Sci 9(10): 474-480

Fries P (2009) Neuronal gamma-band synchronization as a fundamental process in cortical computation. Ann Rev Neurosci 32:209-224

Fries P, Reynolds J H, Rorie AE, Desimone R (2001) Modulation of oscillatory neuronal synchronization by selective visual attention. Science 291:1560-1563

Fries P, Nikolic D, Singer W (2007) The gamma cycle. Trends Neurosci 30(7):309-316

Gruber T, Müller MM, Keil A, Elbert T (1999) Selective visual—spatial attention alters induced gamma band responses in the human EEG. Clin Neurophysiol 110:2074-2085

Hansel D, Mato G, Meunier C (1995) Synchrony in excitatory neural networks. Neural Comput 7(2):307-337

Hayon G, Abeles M, Lehmann D (2005) A model for representing the dynamics of a system of synfire chains. J Comput Neurosci 18: $41-53$
Herz AVM, Gollisch T, Machens CK, Jaeger D (2006) Modeling single-neuron dynamics and computations: a balance of detail and abstraction. Science 314:80-85

Kuhn A, Aertsen A, Rotter S (2004) Neuronal integration of synaptic input in the fluctuation-driven regime. J Neurosci 24(10): 2345-2356

Lachaux JP, Rodriguez E, Martinerie J, Varela FJ (1999) Measuring phase-synchrony in brain signal. Hum Brain Mapp 8:194-208

Latham PE, Richmond BJ, Nelson PG, Nirenberg S (2000) Intrinsic dynamics in neuronal networks. I. Theory. J Neurophysiol 83: $808-827$

Luck SJ, Chelazzi L, Hillyard SA, Desimone R (1997) Neural mechanisms of spatial selective attention in areas V1, V2 and V4 of macaque visual cortex. J Neurophysiol 77:24-72

Lumer ED (2000) Effects of spike timing on winner-take-all competition in model cortical circuits. Neural Comput 12:181-194

Meunier C, Segev I (2001) Neurones as physical objects: structure, dynamics and function. In: Moss F, Gielen S (eds) Handbook of biological physics, vol 4. Elsevier, Amsterdam, pp 353-467

Murthy VN, Fetz FE (1994) Effects of input synchrony on the response of a three-conductance cortical neuron model. Neural Comput 6:1111-1126

Reynolds JH, Desimone R (2003) Interacting roles of attention and visual salience in V4. Neuron 37:853-863

Reynolds J, Chelazzi L, Desimone R (1999) Competitive mechanisms subserve attention in macaque areas V2 and V4. J Neurosci 19(5):1736-1753

Schoffelen JM, Oostenveld R, Fries P (2005) Neuronal coherence as a mechanism of effective corticospinal interaction. Science 308:111-113

Singer W (1999) Neuronal synchrony: a versatile code for the definition of relations? Neuron 24:49-65

Singer W, Gray CM (1995) Visual feature integration and the temporal correlation hypothesis. Annu Rev Neurosci 18:555-586

Taylor K, Mandon S, Freiwald WA, Kreiter AK (2005) Coherent oscillatory activity in monkey area V4 predicts successful allocation of attention. Cereb Cortex 15:1424-1437

Tiesinga PHE (2002) From neuron to brain: statistical physics of the nervous system. In: Macias A, Uribe FJ, Diaz E (eds), Developments in mathematical and experimental physics. Kluwer, Norwell, MA

Tiesinga PHE, Fellous J-M, Jose JV, Sejnowski TJ (2001) Optimal information transfer in synchronized neocortical neurons. Neurocomputing 38-40:397-402

Tiesinga PHE, Fellous J-M, Salinas E, José JV, Sejnowski TJ (2004) Synchronization as a mechanism for attentional gain modulation. Neurocomputing 58-60:641-646

Treue S, Maunsell JHR (1996) Attentional modulation of visual motion processing in cortical areas MT and MST. Nature 382:539-541

von der Malsburg C, Schneider W (1986) A neural cocktail-party processor. Biol Cybern 54(1):29-40

Whittington MA, Traub RD, Kopell N, Ermentrout B, Buhl EH (2000) Inhibition-based rhythms: experimental and mathematical observations on network dynamics. Int J Psychophysiol 38: 315-336

Womelsdorf T, Fries P, Mitra PP, Desimone R (2005) Gamma-band synchronization in visual cortex predicts speed of change detection. Nature 439:733-736

Zeitler M, Fries P, Gielen S (2008) Biased competition through variations in amplitude of gamma-oscillations. J Comp Neurosci 25: 89-107 